\title{
Editorial
}

\section{Girishwar Misra}

Published online: 8 July 2014

(C) National Academy of Psychology (NAOP) India 2014

Notwithstanding the fact that articulating the idea of "good life" has been a perennial concern, the last two decades have evinced increasingly greater interest among psychologists in pursuing investigations about the nature of human well-being and positive human development. Under the banner of 'positive psychology' (PP) dedicated efforts were launched to explicate the dynamics of happiness and satisfaction that are supposed to allow living productive lives.

It may be noted that the ideas about human potential and positive aspects of human strivings were present in the early works of several psychologists including Abraham Maslow and Carl Rogers. For instance, Rogers (1961) talked about the life of 'fully functioning individual' which is 'rich, full and exciting'. According to him good life involves 'the stretching and growing of becoming more and more of one's potentialities'. It represents 'the courage to be'and implies 'launching oneself fully into the stream of life'. Similarly Maslow (1970) had introduced the notion of 'self actualized individuals' and concepts like 'meta motivation', 'B values' and 'peak experience'. In recent years the efforts by Martin Seligman and his colleagues have brought PP at the centre stage. Seligman provided leadership to this movement in year 2000 to supplement the mainstream traditional psychology which was preoccupied with a deficit model of human function and focused on the phenomena of stress, anxiety and depression. There was a clear emphasis on the negative side of human nature.

In the last two decades research in the areas of PP has proliferated on the themes of happiness, resilience, wellbeing, gratitude, flow, hope, human strengths and host of other related constructs. Positive psychological interventions are being designed to augment human strengths in various settings including, health, education, parenting, and work

G. Misra $(\bowtie)$

Department of Psychology, University of Delhi, Delhi 110007, India e-mail: misragirishwar@gmail.com behavior. The factors that contribute the most to a well-lived and fulfilling life are being investigated. The desire to live a happy and an accomplished life is the chief concern of this endeavor.

The field of PP has received encouraging response from researchers in the United States and Western Europe. Their chief concern was mainly with positive emotions, positive character traits, and positive institutions. PP holds that positive emotions, such as joy or interest lead to happiness. They are not simply an indicator that a person is happy. Studying how to cultivate positive emotions, positive psychologists try to discover ways to help people become happier and enjoy flourishing. Its chief endeavor is to promote ways of fostering prosperity and contentment in individuals and communities.

In the course of time a huge amount of intellectual and other resources have been invested into research, publication and conducting Master's and Ph.D. level teaching and research programs in the field of PP. The International Positive Psychology Association (IAPP) has been formed and the field has expanded its application to school, business, health and clinical areas. The developments in PP, however, are primarily situated in the scientific, positivistic and behaviorist model and practices. The discourse of PP largely maintains the individualistic physicalistic and reductionistic stance and neglects the circumstances and world view held by the majority world. It also ignores the issue of sustainability and undermines the role of contextual factors such as culture, ecology and environment.

The move of Indian Psychology (IP) characterizes an indigenous approach which offers a comprehensive perspective to understand the psychological dynamics as well as social reality from a different vantage point. Rooted in the knowledge systems of Indian origin this field conceptualizes mind, its functions and aspects of social relationship in a comprehensive paradigm wherein material and spiritual are integrated. It focuses on the methods and strategies of mind control. It 
advances human functioning in terms of engaging with the process of deconstructing the ego and promoting altruism. It maintains that the search for eternity should be undertaken in the context of deep realization of mortality of body. This makes an enlightened self-understanding as a major concern. It is often reflected in terms life Nirvana and Moksha which are attainable goals while pursuing worldly life. To meet this challenge IP advances the pan human principles and practices of Dharma. They constitute living philosophies of life. Also, IP emphasizes on the use of a first person approach to the study of various issues.

The chief concern of IP, which has a history of several thousand years, is to experience happiness and lead a productive and socially responsible life. The fundamental goal and the academic endeavors of PP and IP seem to share similarity but the diagnosis of (un) happiness and modalities to handle the problems often differ (see Cornelissen, Misra and Varma, 2013; Dalal and Misra, 2010; Rao, Paranjpe and Dalal, 2008).

The similarities and differences between PP and IP raise some interesting questions and issues worth serious research attention. Such an attempt has the potential of collaboration between these two streams of thought. Against this backdrop it becomes essential to explore the arising issues in some detail and organize a dialogue among the colleagues. Professor K.Ramkrishna Rao welcomed the idea and readily agreed to contribute a Target Article for Psychological Studies to initiate the dialogue. He obliged by a piece entitled Positive Psychology and Indian Psychology: In Need for Mutual Reinforcement. This article was circulated among the peer scholars and commentaries were invited. A lively debate followed and we received commentaries from seven scholars who are currently working in the field of IP and have keen interest in PP. They included R.M. Matthijs Cornelissen, Ragubir Singh Pirta, Anand C. Paranjpe, John Christopher, Seema Mehrotra, Kiran K. Salgame and Ajit K. Dalal. We also asked Prof. Rao to respond to the commentaries. He made his response to the commentaries available under the title Indian Psychology in Prospect. I am delighted that Psychological Studies has been able to facilitate this dialogue and is presenting this lively exchange of ideas and debate to its readers. Taken together these reflective contributions raise the concerns, dangers, possibilities and potentials of both PP, IP and the relationship between the two. I express my sincere thanks to all the colleagues who have made this dialogue possible.

This issue of Psychological Studies also contains some other contributions that deal with various aspects of PP. These articles attend to many themes of PP such as happiness, effects of yoga, self awareness, subjective well-being, life satisfaction judgments and positive emotions. They report empirical patterns and reflect the concerns shown in both the traditions.

Indeed, the search for happiness has been a persistent concern among all the civilizations across the globe. The deliberations have indicated two broad thematic categories, namely, a positive state of mind and a vision of life as fulfilling. The relevance of Eastern thought traditions such as Buddhism, Hinduism and the Yoga, Confucianism, and Daoism go beyond a mere hedonistic approach and in diverse ways emphasize the significance and interplay of spirituality, peace, calm mind and harmony. They also indicate the role of balancing (Samatva) and enduring the pain and suffering (Duhkha) as an integral and unavoidable part of life. They also emphasize that pleasure and happiness are not mere synonyms since many pleasures are short lived and have inherent limitations that render them sources of suffering. a distinction is made between pleasures (Preyas) and desirable pleasures (Shreyas).

Cross-cultural as well as cultural studies indicate that the role of agency and communion as two goals of selfhood are articulated in different ways in diverse cultures. The human capacity for adaptation further complicates the experience of happiness and well-being. The aspects of self which help promote happiness are found to vary across cultures. So are the positive and negative manifestations of well-being (see for details Salgame, 2013; Srivastava and Misra, 2011).

The Indian thought goes beyond the empirical self and holds it as a dynamic network of transitory relations at different levels of existence. When the self concerns or egoism are curtailed/reduced we find it easier to focus on the environment and other individuals. What is required is a broad vision that encompasses satisfaction and peace for individuals and those around them. By recognizing a system, of which humans form a part, and acknowledging their interdependence and need for mutual care and reciprocity, IP offers an alternative paradigm. This paradigm helps build a solid disciplinary foundation which may facilitate living a meaningful life in which harmony, coherence and joint pursuit of goals are central.

It is hoped that the dialogue between the traditions of IP and PP that has been initiated by Psychological Studies would continue and yield not only intellectually interesting psychological insights but help evolving strategies and practices to make life on earth enriched, satisfying and healthy.

\section{References}

Cornelissen, R. M. Matthijs, Misra, G. and Varma, S. (Eds.) (2013). Foundations and applications of Indian psychology. New Delhi: Pearson.

Dalal, A. K. and Misra, G. (2010). The core and context of Indian psychology. Psychology and Developing Societies, 22, 121-135.

Maslow, A. (1970). Motivation and personality. New York: Harper \& Row.

Rao, R. K., Paranjpe, A. C., and Dalal, A. K. (Eds.) (2008). Handbook of Indian psychology. New Delhi: Cambridge University Press.

Rogers, C. (1961). On becoming a person. London: Constable. 
Salgame, K. K. (2013). Well-being from the Hindu/Sanatan Dharma perspective. In S. David, I. Boniwell and A.C. Ayers (Eds.), Oxford handbook of happiness (pp. 371-383). New York: Oxford University Press.
Srivastava, A. K. \& Misra, G. (2011). Happiness and well-being: an Indian perspective. In G. Misra (Ed.), Handbook of psychology in India (pp.229-310). New Delhi: Oxford University Press. 